\title{
Effect of PCOS on Omentin-1, Androgens, Biochemical Glycemic and Lipid Profile in Pubertal Girls with Normal Body Mass Index
}

\author{
Burkankulu D', Hassa $\mathbf{H}^{2}$, Aydin $\mathrm{Y}^{3 *}$, Arslantaş $\mathrm{D}^{4}$, \\ Sayıner FD $^{5}$ and Ozerdogan $\mathrm{N}^{5}$ \\ ${ }^{1}$ Medical Doctor, Eskisehir Osmangazi University, \\ Department of Obstetrics and Gynecology, Eskisehir, \\ Turkey \\ ${ }^{2}$ Professor Doctor, Eskisehir Osmangazi University, \\ Department of Obstetrics and Gynecology, Reproductive \\ Medicine Unit, Eskisehir, Turkey \\ ${ }^{3}$ Associate Professor Doctor, Eskisehir Osmangazi \\ University, Department of Obstetrics and Gynecology, \\ Reproductive Medicine Unit, Eskisehir, Turkey \\ ${ }^{4}$ Associate Professor Doctor, Eskisehir Osmangazi \\ University, Department of Public Health, Eskisehir, \\ Turkey \\ ${ }^{5}$ Associate Professor Doctor, Eskisehir Osmangazi \\ University, Nursing College, Eskisehir, Turkey \\ *Corresponding author: Aydin Y, Associate \\ Professor, Eskisehir Osmangazi University School of \\ Medicine, Department of Obstetrics and Gynecology, \\ Reproductive Medicine Unit, Eskisehir, Turkey
}

Received: March 05, 2018; Accepted: April 09, 2018; Published: May 07, 2018

\begin{abstract}
Background: Although the full manifestations of PCOS emerge during adult life, signs are evident during puberty. Understanding the pathophysiological triggers that occur during puberty will benefit the future health of pubertal girls with PCOS.
\end{abstract}

Aim: To investigate the role of Omentin-1 and the serum levels of androgens, glycaemic-lipidemic markers in normal Body Mass Index (BMI) pubertal girls with Polycystic Ovary Syndrome (PCOS) compared with matched controls.

Materials and Methods: In this cross-sectional study we studied with 63 pubertal girls with PCOS and 159 matched controls. PCOS diagnosis was based on the recent ESHRE/ASRM proposal. Indices of insulin sensitivity, metabolic variables, circulating androgen levels, lipidemic markers and serum omentin-1 levels were measured and Blood Pressures (BP) were assessed.

Results: Measurements of low density lipoprotein, triglyceride, insulin, total testosterone, free testosterone, omentin-1 and ratios such as apolipoprotein (apo) B/apoA1 and free androgen index were significantly higher in the study group compared to the control group. BP parameters (systolic/diastolic) were significantly higher in girls with PCOS compared to control group.

Conclusions: The negative cardiovascular effects of PCOS most likely begin as early as puberty, even in the presence of a normal BMI. Long-term cohort studies in pubertal girls with PCOS will improve our knowledge about the pathophysiology and effects of PCOS on cardiovascular and metabolic parameters. We should advise additional preventive strategies other than weight loss to pubertal girls with PCOS.

Keywords: Body mass index; Lipid; Omentin-1; Polycystic ovarian syndrome; Puberty

\section{Introduction}

Polycystic Ovary Syndrome (PCOS) is a common endocrine metabolic disease that occurs in $5-10 \%$ of reproductive aged women [1]. In most cases, PCOS develops during puberty, although some normal pubertal characteristics may overlap with signs of PCOS. This overlap may lead to under-recognition or delayed diagnosis, as symptoms are considered part of normal puberty; conversely, over diagnosis may occur if physiological delay of menses, acne and multimolecular ovaries on ultrasonography are considered diagnostic criteria for PCOS [2]. As proposed in the Amsterdam ESHRE/ASRMSponsored $3^{\text {rd }}$ PCOS Consensus Workshop Group, criteria for the diagnosis of PCOS in adolescents must differ from those used for older women of reproductive age. Specifically, all three Rotterdam criteria must be present to diagnose PCOS in adolescents [3].

PCOS is a pioneering disease for metabolic syndrome, which is mostly associated with central obesity [4]. The endocrine contribution to visceral adipose tissue has now been clearly established; several hormones and cytokines secreted by adipose tissue, termed adipokines, play a role in the pathogenesis of dysfunctional metabolic states such as insulin resistance, cardiovascular disease and metabolic syndrome [5].

Omentin-1 is one of the most important adipokines secreted by visceral adipose tissue. It mainly regulates insulin action and increases Akt phosphorylation in both the absence and presence of insulin in vitro [6]. However, plasma levels of omentin-1 in patients with normal BMI are not as consistent as has been demonstrated in overweight or obese PCOS patients. Akbarzadeh et al. found no significant difference in omentin-1 plasma levels between PCOS patients with normal BMI and non-PCOS patients [7]. Additionally, Choi et al. demonstrated that PCOS-status-dependent differences in omentin-1 level were not significant in women with normal BMI and impaired glucose regulation [8]. Therefore, the association between PCOS and omentin-1 has not been fully elucidated.

Although the full manifestations of PCOS emerge during adult life, signs are evident during puberty [9]. Understanding the pathophysiological triggers that occur during puberty and implementing preventive strategies based on these discoveries will benefit the future health of pubertal girls with PCOS.
Austin J Obstet Gynecol - Volume 5 Issue 5 - 2018

Submit your Manuscript | www.austinpublishinggroup.com

Aydin et al. (@) All rights are reserved
Citation: Burkankulu D, Hassa H, Aydin Y, Arslantaş D, Sayıner FD and Ozerdogan N. Effect of PCOS on Omentin-1, Androgens, Biochemical Glycemic and Lipid Profile in Pubertal Girls with Normal Body Mass Index. Austin J Obstet Gynecol. 2018; 5(5): 1112 
Table 1: Baseline demographic and clinical parameters of the patients.

\begin{tabular}{|c|c|c|c|}
\hline & Patient group $(\mathrm{n}=63)$ & Control group $(\mathrm{n}=159)$ & P value \\
\hline Age (years) & $15.72 \pm 1.20$ & $16.37 \pm 1.84$ & $\mathrm{NS}$ \\
\hline Weight $(\mathrm{kg})$ & $57.28 \pm 9.31$ & $60.37 \pm 11.97$ & $\mathrm{NS}$ \\
\hline Height $(\mathrm{cm})$ & $160.72 \pm 6.52$ & $162.49 \pm 4.87$ & $\mathrm{NS}$ \\
\hline BMI $\left(\mathrm{kg} / \mathrm{m}^{2}\right)$ & $20.23(14.04-24.72)$ & $21.03(15.24-24.09)$ & $\mathrm{NS}$ \\
\hline $\begin{array}{c}\text { Waist } \\
\text { circumference }\end{array}$ & $77.49 \pm 21.36$ & $78.73 \pm 10.22$ & $\mathrm{NS}$ \\
\hline $\begin{array}{c}\text { Systolic blood } \\
\text { pressure } \\
\text { (mmHg) }\end{array}$ & $112.14 \pm 12.64$ & $104.49 \pm 13.93$ & $<0.01$ \\
\hline $\begin{array}{c}\text { Diastolic blood } \\
\text { pressure } \\
\text { (mmHg) }\end{array}$ & $74.12 \pm 9.09$ & $69.01 \pm 11.29$ & $<0.01$ \\
\hline
\end{tabular}

NS: Non-Significant, BMI: Body Mass Index.

To our knowledge, the relationship between omentin-1 and PCOS in pubertal girls has not been previously reported. In this study, we evaluated the role of omentin-1 in pubertal girls with PCOS and normal BMI. We also investigated the effects of PCOS on androgens and on biochemical glycemic and lipidemic markers in this select population.

\section{Materials and Methods}

This cross-sectional case control study was conducted in the Reproductive Medicine Unit of Eskisehir Osmangazi University between October 2012 and November 2013. The study protocol was approved by the Ethics Committee of our University.

We enrolled 63 pubertal girls aged 15-19 years with a BMI less than $25 \mathrm{~kg} / \mathrm{m}^{2}$ who were referred to our center with signs of hirsutism or oligomenorrhea. The diagnosis of PCOS was based on the recent ESHRE/ASRM proposal and required that all three of the Rotterdam criteria for diagnosing PCOS in adolescents be met [3] As recommended by ESHRE/ASRM, the diagnostic criteria included the presence of 1) hyperandrogenemia, defined as elevated blood androgens plus clinical evidence of hyperandrogenemia measured with Ferriman Gallwey score; 2) oligomenorrhea present for 2 years $(<10$ menses a year or at 35 day intervals) or primary amenorrhea at age 16; and 3) an abdominal ultrasound demonstrating an ovarian volume greater than $10 \mathrm{~cm}^{3}$. The control group consisted of 159 pubertal girls matched for age and BMI, all of whom had no history or evidence of oligomenorrhea, hirsutism or acne. Exclusion criteria for both groups were 1) previously diagnosed cardiovascular disease; 2) presence of an endocrine disorder such as thyroid dysfunction or hyperprolactinemia; 3) presence of congenital adrenal hyperplasia, androgen secreting neoplasms or severe insulin resistance; 4) history of any type of drug use, including contraceptives, anti-diabetic, antihypertensive, anti-androgenic or weight reduction agents; 5) history of smoking or alcohol use; 6) BMI higher than $25 \mathrm{~kg} / \mathrm{m}^{2}$.

Clinical variables such as weight, height, and Blood Pressure (BP) were assessed in all subjects using standard protocols during outpatient hospital visits. The BMI was calculated as weight divided by the square of height $\left(\mathrm{kg} / \mathrm{m}^{2}\right)$.

All biochemical and hormonal measurements were obtained during the basal portion of the follicular phase (day 2-5 of menstruation) and were processed in the biochemistry laboratory of University Hospital. Plasma was processed from blood samples by adding $1 \mathrm{mg} / \mathrm{ml} \mathrm{Na2-EDTA}$. The blood samples were centrifuged at $3000 \mathrm{~g}$ for 15 minutes at $4{ }^{\circ} \mathrm{C}$. Immediately after centrifugation, the plasma samples were frozen and stored at $-80^{\circ} \mathrm{C}$ for a period of no more than 4 weeks.

Assays for glucose, insulin, total testosterone and Sex HormoneBinding Globulin (SHBG) were performed using an automated analyzer (Abbott Architect; Abbott Laboratories, Abbott Park, IL). Free testosterone levels were measured with a commercially available ELISA kit (Diasource, Belgium). All lipid parameters (total cholesterol, Triglyceride (TG), high-density lipoprotein cholesterol (HDL-cholesterol), and low-density lipoprotein cholesterol (LDLcholesterol)) were measured by an enzymatic colorimetric test. Apolipoprotein (apo) A-I and B levels were determined by the polyethylene glycol-enhanced immunoturbidimetric assay (Siemens Healthcare Diagnostics Inc.). The plasma level of omentin-1 (BioVendor, Candler, NC and USA) was measured using a commercially available ELISA according to the manufacturer's protocols. The Free Androgen Index (FAI) was calculated as total testosterone/SHBGX100. The estimate of insulin resistance by the Homeostasis Model Assessment (HOMA) score was calculated as fasting insulin $\mathrm{x}$ fasting glucose/22.5. For all hormonal assays, intraand inter-assay Coefficients of Variation (CV) were $<5 \%$.

Statistical analysis was performed using SPSS version 20.0 for Windows (Statistical Package for Social Science, IBM SPSS Inc., Chicago, IL, USA). Descriptive analysis was used to determine the means and standard deviations of the variables. Significant differences between the mean values were estimated by Mann Whitney $U$ test. Statistical significance was considered for $\mathrm{p}<0.05$.

\section{Results}

Table 1 demonstrates the anthropometric, biochemical, and hormonal data for pubertal girls with PCOS and the control group. Control group consisted of 159 pubertal girls matched for age and BMI. BP parameters (systolic/diastolic) were significantly higher in girls with PCOS compared to control group $(112.44 \mathrm{mmHg}$ vs. $104.49 \mathrm{mmHg}$; $<0.001$ for systolic BP, $74.12 \mathrm{mmHg}$ vs. $69.01 \mathrm{mmHg}$; $\mathrm{p}<0.007$ for diastolic BP). There was no significant difference in the Homeostasis Model Assessment Insulin Resistance (HOMA-IR) scores and plasma levels of fasting glucose, total cholesterol, HDL, apo-A1 and SHBG in girls with PCOS compared to the control group (Table 2).

Conversely, girls with PCOS had higher fasting insulin ( $\mathrm{p}=0.007)$, LDL $(\mathrm{p}=0.017)$, triglyceride $(\mathrm{p}=0.045)$, apoB $(\mathrm{p}<0.001)$, total $(\mathrm{p}<0.001)$ and free testosterone $(\mathrm{p}=0.001)$ and omentin- $1(\mathrm{p}=0.018)$ levels compared to control group. Additionally apoB/apoA1 $(\mathrm{p}=0.021)$ and FAI $(\mathrm{p}=0.004)$ values were significantly higher in girls with PCOS compared to the control group (Table 2).

\section{Discussion}

We found that biochemical and hormonal measurements of LDL, $\mathrm{TG}$, insulin, total testosterone, free testosterone and ratios such as apoB/apoA1 and FAI were significantly higher in PCOS adolescents with normal BMI compared to the control group. Importantly, omentin-1 values were also significantly higher in the study group compared to the control group. Additionally, blood pressure 
Table 2: Comparison of omentin-1, androgens and biochemical glycemic and lipid profile in patients.

\begin{tabular}{|c|c|c|c|}
\hline & $\begin{array}{c}\text { Patient group } \\
(n=63)\end{array}$ & $\begin{array}{c}\text { Control group } \\
(n=159)\end{array}$ & P value \\
\hline Fasting insulin $(\mu \mathrm{lU} / \mathrm{ml})$ & 14,35 & 10,93 & 0,007 \\
\hline Fasting glucose $(\mathrm{mg} / \mathrm{dl})$ & 84,44 & 85,91 & $\mathrm{NS}$ \\
\hline HOMA IR & 2,85 & 2,35 & $\mathrm{NS}$ \\
\hline Total cholesterol $(\mathrm{mg} / \mathrm{dl})$ & 156,48 & 153,98 & $\mathrm{NS}$ \\
\hline HDL $(\mathrm{mg} / \mathrm{dl})$ & 61,09 & 57,98 & $\mathrm{NS}$ \\
\hline LDL $(\mathrm{mg} / \mathrm{dl})$ & 92,88 & 86,14 & 0,017 \\
\hline Triglyceride $(\mathrm{mg} / \mathrm{dl})$ & 91,22 & 78,91 & 0,045 \\
\hline ApoB & 97,57 & 69,04 & 0,000 \\
\hline ApoA ${ }_{1}$ & 147,00 & 146,33 & NS \\
\hline APOB/A & 0,69 & 0,47 & 0,021 \\
\hline Total testosterone $(\mathrm{ng} / \mathrm{dl})$ & 45,36 & 28,23 & 0,000 \\
\hline Free testosterone $(\mathrm{pg} / \mathrm{ml})$ & 3,26 & 1,99 & 0,001 \\
\hline SHBG (nmol/liter) & 56,93 & 45,45 & $\mathrm{NS}$ \\
\hline FAl & 5,41 & 2,91 & 0,004 \\
\hline Omentin-1 $(\mathrm{ng} / \mathrm{ml})$ & 503,99 & 478,99 & 0,018 \\
\hline
\end{tabular}

HOMA IR: Homeostasis Model Assessment Insulin Resistance; HDL: HighDensity Lipoprotein Cholesterol; LDL: Low-Density Lipoprotein Cholesterol apoA1: ApolipoproteinA-I; apoB: Apolipoprotein B; SHBG: Sex Hormone-Binding Globulin; FAI: Free Androgen Index.

measurements (both systolic and diastolic) were significantly higher in girls with PCOS compared to controls.

In the INTERHEART study, it was concluded that the apoB/ apoA1 ratio was superior to any of the conventional cholesterol measurements for estimating the risk of acute myocardial infarction in both sexes and for all ages in the general population [10]. Juonale et al. observed that, in adolescents between the ages of 12 and 18, increased baseline apoB/apoA1 ratios indicated predisposition to the development of subclinical atherosclerosis in adulthood [11]. Additionally, the apoB/apoA-I ratio was superior to the LDL/HDL ratio for predicting intima media thickening in adolescence [11]. However, there are few data regarding how PCOS influences the apoB/ apoA1 ratio. To our knowledge, this study is the first to demonstrate an association between the apoB/apoA1 ratio and PCOS in pubertal girls. Our results suggest that, even in the presence of a normal BMI, increased ApoB and decreased ApoA1 levels in girls with PCOS may be an early sign of impending metabolic dysfunction and increased cardiovascular risk.

$\mathrm{BP}$ is one of the risk factors for increased cardiovascular risk. We demonstrated that even if the BMI is normal, girls with PCOS may have higher BP measurements (diastolic BP: $74.12 \pm 9.09$ vs. $69.01 \pm 11.29$; p <0.01, systolic BP: $112.14 \pm 12.64$ vs. $104.49 \pm 13.93$; $<<0.01)$. In a cross sectional study Zachurzok-Buczynska et al. demonstrated significantly higher 24 hour mean and diastolic BP in girls with PCOS compared to the control group [12]. So independent of overweight status, presence of PCOS seems predictive for hypertension and also cardiovascular disease. Consequently, preventive strategies, other than weight loss, designed to lower the risk of developing cardiovascular disease should be considered as early as puberty.

One limitation of our study is that we did not perform a glucose challenge test in participants, so we could not properly identify girls with impaired glucose regulation. As a result, based on the fasting insulin-glucose measurements and HOMA-IR calculations, IR was not present in either group. Further, serum androgen markers (FAI, total and free testosterone) were significantly higher in girls with PCOS. IR is one of the most common findings in obese PCOS patients, with a prevalence of 50-75\%. Hyperinsulinemia may contribute to hyperandrogenism through increased ovarian production of androgens and decreased production of SHBG. This may be a probable cause of hyperandrogenemia with the absence of IR in our study population with normal BMI. Moreover, as Baillargeon and Nestler proposed, even in the absence of insulin resistance, the ovarian response to insulin may be abnormal in PCOS patients [13] and insulin resistance may be a modifying rather than a causative factor for hyperandrogenemia [14].

There are a number of reports about the association of PCOS and omentin-1. Of particular interest is the finding by Tan et al., who reported significantly lower levels of omentin-1 mRNA and protein in the omental adipose tissue of women with PCOS, in addition to decreased plasma omentin-1 levels in overweight PCOS patients of reproductive age [15]. Mahde et al. also found decreased omentin-1 levels in obese PCOS patients and further suggested that lower omentin-1 levels are predictive of the metabolic dysfunction associated with PCOS [16]. In contrast, Akarbarzadeh et al. found that omentin-1 was not lower in normal BMI PCOS patients compared to controls [7]. Our results differ from other studies in that we found omentin-1 levels to be higher in PCOS pubertal girls with normal BMI when compared to controls. There may be various causes for our controversial results. Firstly, our study population of pubertal girls with normal BMI differed from the populations of other studies, and the mechanisms underlying the regulation of omentin-1 may be completely different in this young age group. Secondarily, a normal BMI may contribute to increased omentin-1 levels in the pubertal girls considered in this study. Moreover, the absence of IR in our study population may also contribute to increased omentin-1 levels. Consistent with the results presented by Choi et al. [8] and Mahde et al. [16], we propose that omentin-1 regulation is multifactorial and dependent on mechanisms other than BMI and IR that have yet to be identified, at least in pubertal girls with normal BMI. Lastly, higher omentin-1 levels, which are suggested to be a cardioprotective adipokine [17], may be a result of compensatory mechanism and increase of the BMI in this risky PCOS population may result with a decrease in omentin-1 in adult period. But only way to confirm this speculation is to observe our population in a long period of time. So, long-term studies of girls with PCOS that begin during puberty will give us more knowledge about the regulation and effect of omentin-1 in PCOS.

\section{Conclusion}

The negative cardiovascular effects of PCOS most likely begin as early as puberty, even in the presence of a normal BMI. The association of PCOS and omentin-1 is more complex than previously thought and appears to regulate metabolism differently during puberty. Longterm cohort studies in pubertal girls with PCOS will improve our knowledge about the pathophysiology and effects of PCOS. Lastly, we must advise additional preventive strategies other than weight loss to pubertal girls with PCOS. 


\section{Authors' Contribution to the Manuscript}

Burkankulu D: Data collection or management

Hassa H: Protocol/project development

Aydin Y: Protocol/project development, Data analysis, Manuscript writing/editing

Aslantas D: Protocol/project development, Data analysis, Data collection or management, Manuscript writing/editing

Sayiner D: Protocol/project development, Data analysis, Data collection or management

Ozerdogan N: Protocol/project development, Data analysis, Data collection or management

\section{Compliance with Ethical Standards}

Funding: This study was partially supported by Eskisehir Osmangazi University Scientific Research Project Commission, Project No. 201211008.

Ethical approval: All procedures performed in studies involving human participants were in accordance with the ethical standards of the institutional and/or national research committee and with the 1964 Helsinki declaration and its later amendments or comparable ethical standards.

Informed consent: Informed consent was obtained from all individual participants included in the study.

\section{References}

1. Dunaif A. Insulin resistance and the polycystic syndrome: mechanism and implications for pathogenesis. Endocr Rev. 1997; 18: 774-778.

2. Hardy TS, Norman RJ. Diagnosis of adolescent polycystic ovary syndrome Steroids. 2013; 78: 751-757.

3. Fauser BC, Tarlatzis BC, Rebar RW, Legro RS, Balen AH, Lobo R, et al Consensus on women's health aspects of polycystic ovary syndrome (PCOS): the Amsterdam ESHRE/ASRM-Sponsored 3rd PCOS Consensus Workshop Group. Fertil Steril. 2012; 97: 28-35

4. Rossi B, Sukalich S, Droz J, Griffin A, Cook S, Blumkin A, et al. Prevalence of metabolic syndrome and related characteristics in obese adolescents with and without polycystic ovary syndrome. J Clin Endocrinol Metab. 2008; 93: 4780-4785.
5. Kershaw EE, Flier JS. Adipose tissue as an endocrine organ. J Clin Endocrino Metab. 2004; 89: 2548-2552.

6. Yang RZ, Lee MJ, Hu H, Pray J, Wu HB, Hansen BC, et al. Identification of omen tin as a novel depot-specific adipokine in human adipose tissue: Possible role in modulating insulin action. Am J Phys Endocrinol Metab. 2006; 290: 1253-1257.

7. Akbarzadeh S, Ghasemi S, Kalantarhormozi M, Nabipour I, Abbasi F, Aminfar A, et al. Relationship among plasma adipokines, insulin and androgens level as well as biochemical glycemic and lipidemic markers with incidence of PCOS in women with normal BMI. Gynecol Endocrinol. 2012; 28: 521-526.

8. Choi JH, Rhee EJ, Kim KH, Woo HY, Lee WY, Sung KC. Plasma omentin-1 levels are reduced in non-obese women with normal glucose tolerance and polycystic ovary syndrome. Eur J Endocrinol. 2011; 165: 789-794.

9. Norman RJ, Dewailly D, Legro RS, Hickey TE. Polycystic ovary syndrome. Lancet. 2007; 370: 685-690

10. McQueen MJ, Hawken S, Wang X, Ounpuu S, Sniderman A, Probstfield J, et al. Lipids, lipoproteins, and apolipoproteins as risk markers of myocardia infarction in 52 countries (the INTERHEART study): a case-control study. Lancet. 2008; 372: 224-229.

11. Juonala M, Viikari JS, Kähönen $M$, Solakivi $T$, Helenius $H$, Jula $A$, et al. Childhood levels of serum apolipoproteins $B$ and $A-I$ predict carotid intima-media thickness and brachial endothelial function in adulthood: the cardiovascular risk in young Finns study. J Am Coll Cardiol. 2008; 52: 293298.

12. Zachurzok-Buczynska A, Szydlowski L, Gawlik A, Wilk K, Malecka-Tendera E. Blood pressure regulation and resting heart rate abnormalities in adolescent girls with polycystic ovary syndrome. Fertil Steril. 2011; 96: 1519-1525.

13. Baillargeon JP, Nestler JE. Commentary: polycystic ovary syndrome: a syndrome of ovarian hypersensitivity to insulin? J Clin Endocrinol Metab. 2006; 91: 22-28.

14. Legro RS, Driscoll D, Strauss JF III, Fox J, Dunaif A. Evidence for a genetic basis for hyperandrogenemia in polycystic ovary syndrome. Proc Natl Acad Sci USA. 1998; 95: 149-156.

15. Tan BK, Heutling D, Chen J, Farhatullah S, Adya R, Keay SD, et al. Metformin decreases the adipokine vaspin in overweight women with polycystic ovary syndrome concomitant with improvement in insulin sensitivity and a decrease in insulin resistance. Diabetes. 2008; 57: 1501-1506.

16. Mahde A, Shaker M, Al-mashhadani Z. Study of omentin-1 and other adipokines and hormones in PCOS patient. OMJ. 2009; 24: 108-112.

17. Greulich S, Chen WJ, Maxhera B, Rijzewijk LJ, van der Meer RW, Jonker JT, et al. Cardioprotective properties of omentin-1 in type 2 diabetes: evidence from clinical and in vitro studies. PLoS One. 2013; 8: e59697.
Austin J Obstet Gynecol - Volume 5 Issue 5 - 2018 Submit your Manuscript | www.austinpublishing group.com Aydin et al. (C) All rights are reserved
Citation: Burkankulu D, Hassa H, Aydin Y, Arslantas D, Sayıner FD and Ozerdogan N. Effect of PCOS on Omentin-1, Androgens, Biochemical Glycemic and Lipid Profile in Pubertal Girls with Normal Body Mass Index. Austin J Obstet Gynecol. 2018; 5(5): 1112. 